\title{
An Untoward Effect: Anticoagulation Therapy in Cancer Patients Leads to Gastrointestinal Bleeding and Increased Mortality
}

\author{
Dr. Arine Mathew, MBBS, MD
}

\section{Introduction}

Cancer, a condition involving uncontrolled division of cells, ranks second among leading causes of death worldwide. ${ }^{[1]}$ According to an estimate, cancer will contribute 609,640 deaths in the year 2018 in United States. ${ }^{[2]}$ Therefore, cancer is a major public concern as it has high morbidity and mortality rates. The patients with cancer are at high risk of developing venous thromboembolism (VTE) as it is a hypercoagulable state. ${ }^{[3]}$ Therefore, thrombotic events are a major cause of morbidity and mortality among the patients with cancer. ${ }^{[4,5]}$ Recommendations of anticoagulation in acute VTE are same in the patients with or without cancer. ${ }^{[3]}$ Anticoagulation prophylaxis and therapy is recommended in the patients with cancer who are hospitalized with acute illness or undergoing major surgery and those who have developed VTE, respectively. ${ }^{[6]}$ Usually, low molecular weight heparin (LMWH), vitamin $\mathrm{K}$ antagonist (VKA) and direct oral anticoagulants are used for the treatment of VTE in the patients with cancer. The purpose of this anticoagulation is to prevent extension, recurrence and embolism of thrombus. However, higher recurrence of VTE and risk of bleeding complicates the treatment.

Gastrointestinal (GI) bleeding is a major concern of patients receiving anticoagulation therapy as treatment or prophylaxis. As anticoagulation therapy for the treatment or prophylaxis of VTE in the patients with or without cancer is same, GI bleeding in the patients with malignancy may complicate the management. In this paper, we have reviewed the available literature on GI bleeding and mortality among the patients with cancer who received anticoagulation therapy.

\section{Search Strategy}

An online search was conducted on PubMed, Embase and Google Scholar using keywords "Anticoagulation Therapy", "Coagulopathy", "Cancer", "Malignancy", "Gastrointestinal Bleeding", "Mortality", and combination of these words to find related peer-reviewed articles. The collected included only original articles and meta-analyses published in peer-review journals.

\section{Literature Review}

Anticoagulation therapy in the patients with malignancy is associated with side effects, especially prolonged bleeding or heavy bleeding. Excessive bleeding secondary to anticoagulation therapy in cancer patients may exceed the benefits for which the therapy is given. As mentioned above, low molecular weight heparin (LMWH), vitamin $\mathrm{K}$ antagonist (VKA) and direct oral anticoagulants are used for the prophylaxis or treatment of VTE in the patients with malignancy. This paper reviews the literature on anticoagulation therapy in cancer patients in terms of gastrointestinal bleeding and increased mortality.

Low Molecular Weight Heparin (LMWH): Heparin is an anticoagulant or blood thinner drug. Produced by chemical or enzymatic fractionation or depolymerisation of unfractionated heparin (UFH), LMWH are small chains or product of heparin. ${ }^{[7]}$ LMWH cause confirmation change by binding to anti-thrombin (AT), leading to inhibition of conversion of activated X (factor Xa) which in turn blocks the conversion of prothrombin to thrombin. Dalteparin, Enoxaparin and Tinzaparin are some common LMWH.

LMWH is an acceptable intervention for the treatment of thrombosis in cancer patients. In a study by Klil-Drori et al. ${ }^{[9]}$ compared LMWH with VKAs in the patients on dialysis in terms of risk of major bleeding while treating VTE. They reported improved effectiveness with LMWH without increasing the risk of major bleeding as compared to VKAs. CLOT study reveals that Dalteparin is more effective in reducing the risk of recurrent thromboembolism and safer in the patients with cancer as compared to those who use conventional anticoagulation therapy. ${ }^{[8]}$ However, there was no significant difference between Dalteparin and VKA in terms of major bleeding and mortality rate. On the contrast, Khorana et al., ${ }^{[10]}$ conducted a multicentre randomized controlled trial (RCT) enrolling 98 patients with Khorana score > 3 in order to determine the effect of Dalteparin in preventing VTE and risk factors. They reported that Dalteparin did not reduce the risk of VTE significantly while it increased risk of clinically relevant bleeding.

Enoxaparin is also effective in the prophylaxis and treatment of VTE in the patients with cancer. ${ }^{[1]}$ Enoxaparin and Cancer (ENOXACAN) II was the pioneer study that documented benefits of Enoxaparin in the prophylaxis of VTE in the cancer patients who undergo surgery. ${ }^{[12]}$ Two important studies have assessed the efficacy of Enoxaparin in the treatment of VTE in cancer patients. CANTHANOX is an RCT, which included 146 patients with cancer in order to compare Enoxaparin with Warfarin in terms of the treatment and secondary prophylaxis of VTE. ${ }^{[13]}$ It reported that $21.1 \%$ patients in the Warfarin group and $10.5 \%$ patients in the Enoxaparin group experienced major bleeding. It also reported that six patients died of hemorrhage in the Warfarin group with no death in the Enoxaparin group. The study concluded that Warfarin is associated with high risk of major bleeding in the patients with 


\section{International Journal of Innovative Research in Medical Science (IJIRMS) Volume 03 Issue 12 Dec 2018, ISSN: 2455-8737, Imp. Factor - 4.102 Available online at - www. ijirms.in}

VTE and cancer as compared to Enoxaparin. Similarly, ONCENOX trial included 101 patients with cancer and acute VTE in order to compare Enoxaparin with Warfarin in terms of the treatment and secondary prophylaxis of VTE. ${ }^{[14]}$ It reported recurrent VTE in $10.5 \%$ and $5.1 \%$ patients in Warfarin and Enoxaparin groups, respectively. ONCENOX trial favored Enoxaparin in terms of feasibility, affectivity and tolerability. However, there were no events of fatal hemorrhage or difference of mortality in both the groups. It does not mean that Enoxaparin does not cause hemorrhage in the patients with cancer. Enoxaparin may cause gastrointestinal or major bleeding in the extended treatment for VTE, but less than that with VKAs. ${ }^{[10]}$

Tinzaparin is another LMWH, effective for the treatment of VTE in the patients with cancer. However, major bleeding associated with Tinzaparin use in onclology is similar to those with VKAs. ${ }^{[15]}$

Vitamin K Antagonist (VKA): Vitamin K, a fat-soluble vitamin, serves as a co-factor for coagulation proteins II, VII, IX and X. VKAs include warfarin, acenocoumarol, phenprocoumon and fluindione. Warfarin has been widely studied and frequently used VKA worldwide. VKAs work by inhibiting vitamin $\mathrm{K}$ epoxide reductase - a vitamin $K$ reducing enzyme ${ }^{[16]}$. They are used for long-term oral anticoagulation therapy in the patients with cancer and VTE. However, hemorrhage or bleeding is the most common side effect of VKAs.

Chen et al., ${ }^{[17]}$ retrospectively studied the incidence of GI bleeding in 401 Warfarin-antocoagulated patients. They reported incidence of GI bleeding in $3.9 \%$ per patients-years. They also reported that $27.3 \%$ patients experienced rebleeding after restarting Warfarin, and $16,7 \%$ patients experienced VTE after the cessation of Warfarin. They reported mortality in 14 patients with GI bleeding where two patients died solely due to heavy bleeding. It shows that Warfarin is associated with significant risk of GI bleeding which may affect the clinical outcome and mortality. Studies have reported that the cancer patients on Warfarin therapy experience major GI bleeding. As mentioned above, CANTHANOX trial has reported major GI bleeding with fatal outcome in the patients who received Warfarin. ${ }^{[13]}$ Flack et al. ${ }^{[18]}$ reported that major GI bleeding was observed with Warfarin in the patients with gastric cancer and with Debigatrin in the patients with colorectal cancer. An interesting thing is that GI bleeding as side effect of anticoagulation increases the rate of cancer detection. ${ }^{[19]}$

Cangemi et al., ${ }^{[20]}$ identified 803 patients on non-vitamin $\mathrm{K}$ antagonist oral anticoagulants (NOACs) and 6,263 on warfarin through VA pharmacy database in order to compare the rate of GI bleeding. They reported GI bleeding in $2.5 \%$ and $0.5 \%$ patients on Warfarin and NOACs, respectively. This study revealed that GI bleeding is four times more in the patients who receive Warfarin therapy as compared to those who receive NOACs. Warfarin therapy reduces the risk of VTE but increases the risk of GI bleeding and mortality in the patients with malignancy. In this regard, Chen et al., ${ }^{[17]}$ reported mortality in two Warfarinanticoagulated patients due to uncontrolled bleeding. Charlton et al., ${ }^{[21]}$ studied Warfarin, Dabigatran and Rivaroxaban in terms of hospital stay and mortality for bleeding. They reported all-cause mortality of $7.1 \%, 8 \%$ and $4.6 \%$ with Warfarin, Dabigatran and Rivaroxaban, respectively after 30 days of being discharged. Similarly, they reported all-cause mortality of $9.2 \%, 9.7 \%$ and $5 \%$ with Warfarin, Dabigatran and Rivaroxaban, respectively after 90 days of being discharged. In a meta-analysis, Zhang et al., ${ }^{[2]}$ reviewed 2185 patients suffering from lung cancer and at high risk of developing VTE, who were offered adjunctive anticoagulation with VKAs or subcutaneous heparin without indication of anticoagulation. Although they reported survival benefit with adjunctive anticoagulation; however, incidence of bleeding risk was increased with the use of VKAs and subcutaneous heparin.

Direct Oral Anticoagulants (DOACs): The advent of DOACs has revolutionized the anticoagulation therapy, providing a novel treatment option. DOACs (Dabigatran, Rivaroxaban, Apixaban and Edoxaban) directly inhibit thrombin or factor Xa. These newly developed drugs possess comparable efficacy and reduced adverse effects such as major bleeding or GI bleeding as compared to Warfarin in terms of venous thromboembolism (VTE) management. ${ }^{[23]} \mathrm{Li}$ et al., ${ }^{[24]}$ reviewed DOACs and LMWH for the treatment of VTE in cancer patients. They reported that DOACs were more effective than LMWH, but were associated with risk of major bleeding. Hence, whatever the anticoagulant group of drugs is, bleeding risk is increased. Studies have revealed that anticoagulation in cancer patients increases 2-6 folds higher risk of bleeding. ${ }^{[25]}$

Chai-Adisaksopha et al., ${ }^{[26]}$ studied mortality outcome with DOACs conducting a meta-analysis including 13 RCTs involving 102707 adult patients. They reported lowered rate of fatal bleeding and case-fatality rate of major bleeding (7.57\%) with DOACs as compared to those with Warfarin (11.04\%). In another study, DOACs may increase up to $25 \%$ GI bleeding as compared to that with VKAs. ${ }^{[27]}$ In this context, although DOACs have lower risk of major bleeding and case-fatality rates; however, management of bleeding is challenging due to non-availability of their antidotes. Yhim et al. ${ }^{[28]}$ conducted a prospective, multicenter, open-label trial including 124 patients with active cancer undergoing chemotherapy or radiotherapy in order to determine the outcome of long-term use rivaroxaban for the treatment of VTE. They followed the study for 6 months and reported major bleeding in $5.3 \%$ cases. They reported 28 deaths during the study where $10.7 \%$ contribution was added by fatal bleeding. Although DOACs are feasible to use and more effective than other anticoagulants; however, bleeding related deaths may worsen the outcome.

\section{Conclusion}

Gastrointestinal bleeding is an important and most common untoward effect of anticoagulation therapy in the patients with malignancy, which may increase mortality. VKAs rank high in terms of GI bleeding in the patients with cancer. DOACs are also associated with major GI bleeding but less than that with Warfarin. However, mortality rate is comparable in LMWH, VKAs and DOACs. Therefore, judicious selection of the patients with cancer for anticoagulation therapy is of prime importance in order to avoid fatal consequences. As far as mortality is concerned, the role of anticoagulation in cancer patients still needs to be evaluated thoroughly.

\section{References}

[1] Nagai H, Kim YH. Cancer prevention from the perspective of global cancer burden patterns. J Thorac Dis 2017; 9(3): 448-51. 


\section{International Journal of Innovative Research in Medical Science (IJIRMS) Volume 03 Issue 12 Dec 2018, ISSN: 2455-8737, Imp. Factor - 4.102 Available online at - www. ijirms.in}

[2] Siegel RL, Miller KD, Jemal A. Cancer statistics, 2018. CA Cancer J Clin 2018; 68(1): 7-30.

[3] Bauer KA. Treatment of venous thromboembolism in patients with malignancy [Internet]. Jul 13, 2018 [cited August 26, 2018]. Available from: https://www.uptodate.com/contents/treatment-of-venousthromboembolism-in-patients-with-malignancy.

[4] Eichinger S. Cancer associated thrombosis: risk factors and outcomes. Thrombos Res 2016; 140(1): S12-7.

[5] Shah S, Datta YH, Norby F, Alonso A. Direct oral anticoagulants in patients with cancer. Blood 2016; 128(22): 877

[6] Khorana AA, Carrier M, Garcia DA, Lee AYY. Guidance for the prevention and treatment of cancerassociated venous thromboembolism. J Thromb Thrombolysis 2016; 41: 81-91.

[7] Gray E, Mulloy B, Barrowcliffe TW. Heparin and lowmolecular-weight heparin. Thromb Haemost 2008;99:807-18.

[8] Linkins LA. Management of venous thromboembolism in patients with cancer: role of dalteparin. Vasc Health Risk Manag 2008;4(2):279-87.

[9] Lee AY, Levine MN, Baker RI, Bowden C, Kakkar AK, Prins $\mathrm{M}$, et al. Randomized Comparison of LowMolecular-Weight Heparin versus Oral Anticoagulant Therapy for the prevention of recurrent venous thromboembolism in patients with cancer (CLOT) investigators. Low-molecular-weight heparin versus a coumarin for the prevention of recurrent venous thromboembolism in patients with cancer. $\mathrm{N}$ Engl $\mathrm{J}$ Med 2003; 349(2):146-53.

[10] Klil-Drori AJ, Coulombe J, Nessim SJ, Tagalakis V. The risk of major bleeding with low-molecular-weightheparins for venous thromboembolism in dialysis patients: The Q-VTE Study. Blood 2016; 128:89.

[11] Qureshi W, Ali Z, Amjad W, Alirhayim Z, Farooq H, Qadir S, Khalid F, et al. Venous thromboembolism in cancer: an update of treatment and prevention in the era of newer anticoagulants. Front Cardiovasc Med 2016; 3:24.

[12] Bergqvist D, Agnelli G, Cohen AT, Eldor A, Nilsson PE, Le Moigne-Amrani A, et al. Duration of prophylaxis against venous thromboembolism with enoxaparin after surgery for cancer. N Engl J Med 2002; 346 (13):975-80.

[13] Meyer G, Marjanovic Z, Valcke J, Lorcerie B, Gruel Y, Solal-Celigny P, et al. Comparison of low-molecularweight heparin and warfarin for the secondary prevention of venous thromboembolism in patients with cancer: a randomized controlled study. Arch Intern Med 2002; 162(15):1729-35.

[14] Deitcher SR, Kessler CM, Merli G, Rigas JR, Lyons RM, Fareed J. Secondary prevention of venous thromboembolic events in patients with active cancer: enoxaparin alone versus initial enoxaparin followed by warfarin for a 180-day period. Clin Appl Thromb Hemost 2006; 12(4):389-96.

[15] Dimakakos EP, Vathiotis I, Syrigos K. The Role of Tinzaparin in Oncology. Clin Appl Thromb Hemost 2018; 24(5):697-707.

[16] Ufer M. Comparative pharmacokinetics of vitamin $\mathrm{K}$ antagonists: warfarin, phenprocoumon and acenocoumarol. Clin Pharmacokinet 2005;44(12):122746.

[17] Chen WC, Chen YH, Hsu PI, Tsay FW, Chan HH, Cheng JS, et al. Gastrointestinal hemorrhage in warfarin anticoagulated patients: incidence, risk factor, management, and outcome [Internet]. May 29, 2014 [cited Aug 30, 2018]. Available from: https://doi.org/10.1155/2014/463767.

[18] Flack KF, Desai J, Kolb JM, Chatterjee P, Wallentin LC, Ezekowitz M, et al. Major gastrointestinal bleeding often is caused by occult malignancy in patients receiving warfarin or dabigatran to prevent stroke and systemic embolism from atrial fibrillation. Clin Gastroenterol Hepatol 2017;15(5):682-90.

[19] Asiimwe A, Li JJ, Weerakkody G, Vangerow H, Delisle $\mathrm{F}$, Benoit K, et al. Diagnoses of gastrointestinal cancers after gastrointestinal bleeding in patients receiving clopidogrel or warfarin. Curr Drug Saf 2013;8(4):261-9.

[20] Cangemi DJ, Krill T, Weideman R, Cipher DJ, Spechler $\mathrm{SJ}^{1}$, Feagins LA. A comparison of the rate of gastrointestinal bleeding in patients taking non-vitamin $\mathrm{k}$ antagonist oral anticoagulants or warfarin. Am J Gastroenterol 2017;112(5):734-9.

[21] Charlton B, Adeboyeje G, Barron JJ, Grady D, Shin J, Redgerg RF. Length of hospitalization and mortality for bleeding during treatment with warfarin, dabigatran, or rivaroxaban. PLoS One 2018;13(3):e0193912.

[22] Zhang J, Zhang YL, Ma KX, Qu JM. Efficacy and safety of adjunctive anticoagulation in patients with lung cancer without indication for anticoagulants: a systematic review and meta-analysis. Thorax 2013;68(5):442-50.

[23] Mantha S, Ansell J. Indirect comparison of dabigatran, rivaroxaban, apixaban and edoxaban for the treatment of acute venous thromboembolism. J Thromb Thrombolys 2014;37(2):76-9.

[24] Li A, Garcia DA, Lyman GH, Carrier M. Direct oral anticoagulant (DOAC) versus low-molecular-weight heparin (LMWH) for treatment of cancer associated thrombosis (CAT): A systematic review and metaanalysis. Thromb Res 2018. pii: S0049-3848(18)302160 .

[25] Short NJ, Connors JM. New Oral Anticoagulants and the Cancer Patient. Oncologist 2014;19(1):82-93.

[26] Chai-Adisaksopha C, Hillis C, Isayama T, Lim W, Iorio A, Crowther M. Mortality outcomes in patients receiving direct oral anticoagulants: a systematic review and metaanalysis of randomized controlled trials. J Thromb Haemost 2015;13(11):2012-20.

[27] Deutsch D, Boustiere C, Ferrari E, Albaladejo P, Morange PE, Renamouzig R. Direct oral anticoagulants and digestive bleeding: therapeutic management and preventive measures. Therap Adv Gastroenterols 2017;10(6):495-505.

[28] Yhim HY, Choi WI, Kim SH, Nam SH, Kim KH, Mun $\mathrm{YC}$, et al. Long-term rivaroxaban for the treatment of acute venous thromboembolism in patients with active cancer in a prospective multicenter trial. Korean J Intern Med 2018. doi: 10.3904/kjim.2018.097. [Epub ahead of print] 